\title{
Centralisation et décentralisation dans le Service national de santé : le cas de la médecine de ville
}

Centralisation and Decentralisation in the National Health Service: the Case of

Primary Care

\section{Anémone Kober-Smith}

\section{(2penEdition}

Journals

\section{Édition électronique}

URL : http://journals.openedition.org/rfcb/3445

DOI : $10.4000 /$ rfcb.3445

ISSN : 2429-4373

Éditeur

CRECIB - Centre de recherche et d'études en civilisation britannique

\section{Édition imprimée}

Date de publication : 1 avril 2005

ISBN : 2-911580-20-6

ISSN : 0248-9015

Référence électronique

Anémone Kober-Smith, «Centralisation et décentralisation dans le Service national de santé : le cas de la médecine de ville », Revue Française de Civilisation Britannique [En ligne], XIII-2 | 2005, mis en ligne le 01 avril 2005, consulté le 10 avril 2020. URL : http://journals.openedition.org/rfcb/3445 ; DOI : https://doi.org/10.4000/rfcb.3445

Ce document a été généré automatiquement le 10 avril 2020.

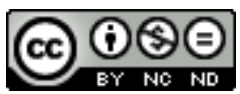

Revue française de civilisation britannique est mis à disposition selon les termes de la licence Creative Commons Attribution - Pas d'Utilisation Commerciale - Pas de Modification 4.0 International. 


\section{Centralisation et décentralisation dans le Service national de santé : le cas de la médecine de ville}

Centralisation and Decentralisation in the National Health Service: the Case of

Primary Care

Anémone Kober-Smith

1 Depuis sa création en 1948, le service national de santé britannique (National Health Service) se caractérise par une forte centralisation administrative de type bureaucratique associée à la position dominante de la profession médicale. Ce n'est qu'à partir des années 1980 que l'on assiste, dans ce secteur comme dans d'autres, à un véritable transfert de pouvoirs du centre vers le niveau local qui s'inscrit dans un mouvement plus large, la nouvelle gestion publique (New Public Management). Dépassant le contexte national, ce courant est fondé sur le principe qu'une gestion inspirée du privé améliore à la fois l'efficience et l'efficacité des services publics ${ }^{1}$. Dans le service de santé, la première manifestation de la nouvelle gestion publique se traduit par l'introduction de pratiques managériales telles que les indicateurs de performance, l'externalisation des services auxiliaires et la délégation budgétaire au niveau des services. Dès le début des années 1990, la réforme se radicalise et la déconcentration des pouvoirs s'intensifie lors de la création d'un quasi-marché qui sépare les producteurs de soins, les établissements, des acheteurs, les autorités sanitaires et certains médecins généralistes. En quelques années, la mutation est si complète que certains auteurs avancent que la nouvelle gestion publique a atteint un stade de maturité dans le $\mathrm{NHS}^{2}$.

2 L'arrivée des travaillistes au pouvoir en 1997 signale un changement de rhétorique sur la réforme du service public. Les notions de marché et de consommateur tendent à être remplacées par celles de citoyen et de partenariat. À partir de 2000, le terme de " localisme " apparaît. Il s'agit d'encourager une nouvelle forme de gouvernance locale en réseaux à laquelle le public participe (networked community governance) ${ }^{3}$. Dans ce modèle, un nouveau principe, la démocratisation du service, s'ajoute à ceux qui 
existaient déjà, l'implantation locale des opérations du marché et la dévolution des pouvoirs vers les cadres de proximité ${ }^{4}$. Pour certains analystes, ce nouveau type de gouvernance reflète les attentes des citoyens d'aujourd'hui pour qui l'efficience ne suffit plus et qui souhaitent faire entendre leur " voix » sur la question des services publics 5 .

Dans la réalité, la politique travailliste est plus ambivalente. D'une part, on assiste à la mise en place de nouvelles agences indépendantes qui exercent une surveillance sur le niveau local par le biais de normes et d'inspection. D'autre part, des réformes décentralisatrices telles que la création de nouvelles fondations hospitalières (Foundation Hospitals), le partenariat financier entre le privé et le public (Public Private Partnership) pour la construction d'hôpitaux et l'autonomie accordée aux nouvelles fondations de soins primaires ${ }^{6}$ (Primary Care Trusts) indiquent que l'on assiste à un renforcement de la déconcentration des pouvoirs entamée par l'administration précédente. Certains observateurs ${ }^{7}$ avancent que cette tension entre flux centralisateur et décentralisateur est déséquilibrée car les nouvelles formes de gouvernance locale sont trop faibles pour remettre en question le modèle managérial, enraciné dans le secteur sanitaire depuis vingt ans. Afin de tester cette hypothèse, nous nous proposons d'examiner dans quelle mesure le discours travailliste sur le «localisme » s'est traduit dans le NHS par une nouvelle forme de gouvernance au niveau local, et plus particulièrement dans les fondations de soins primaires qui regroupent tous les cabinets de médecins généralistes, les infirmières, les pharmaciens et les opticiens d'une localité. Nous tenterons d'évaluer le type et le degré de déconcentration administrative et de participation citoyenne des Primary Care Trusts: s'agit-il uniquement d'une dévolution gestionnaire qui s'inscrit dans le modèle de nouvelle gestion publique, d'une gouvernance trans-sectorielle à laquelle les usagers sont associés ou d'un stade intermédiaire? Dans le service de santé, les fondations de soins primaires forment l'exemple le plus développé de structures décentralisées mises en place par les néo-travaillistes et leur implantation géographique locale, tout comme l'obligation qui est faite aux patients de consulter leur généraliste avant d'accéder au secteur hospitalier secondaire ${ }^{8}$, nous permet de supposer qu'elles sont plus à même que d'autres d'avoir mis en place des formes de participation citoyenne.

Dans un premier temps, nous ferons un bref rappel de la politique sanitaire des conservateurs entre 1979 et 1997 en nous intéressant plus particulièrement au cas des médecins généralistes gestionnaires. Après une rapide présentation des principales réformes travaillistes pour le NHS, nous étudierons l'origine et la mise en place des Primary Care Trusts. Enfin, nous analyserons le degré de déconcentration de ces structures et tenterons de déterminer dans quelle mesure elles associent le public à leurs décisions. Pour ce dernier point, deux aspects seront examinés : les formes de représentation des usagers et leur participation au choix et à l'achat de soins hospitaliers, une des fonctions principales des nouvelles fondations.

\section{Centralisation et marché local sous les conservateurs}

5 La période conservatrice se caractérise par une politique radicale de maîtrise des dépenses et d'amélioration de l'efficacité du service de santé. Ce programme s'accompagne d'une déconcentration de type managérial, qui se manifeste notamment par un transfert des responsabilités gestionnaires vers les établissements et une 
implantation locale de l'achat et de l'offre de services lors de la transformation du service de santé en marché. En parallèle, on assiste à une centralisation accrue qui se traduit par l'affaiblissement des niveaux administratifs intermédiaires et le renforcement de la surveillance exercée par le centre sur le local. Dans les années 1980, cette politique se traduit essentiellement par l'introduction de principes managériaux dans les hôpitaux. Une première réforme d'envergure vise à remplacer les anciennes équipes directoriales mixtes, qui associaient médecins, infirmières et cadres administratifs, par des postes de directeurs généraux (chief executives) créés à chaque niveau du service (établissement, district et région) ${ }^{9}$. Ouverts à la concurrence extérieure et de durée déterminée, ces postes concentrent les pouvoirs entre les mains du directeur hospitalier, qui répond de ses actes devant le niveau supérieur. D'une façon générale, le volume et la précision des données financières et cliniques en provenance des établissements augmentent.

6 À l'intérieur même des établissements, on assiste à un transfert des responsabilités gestionnaires du niveau directorial vers les services qui se traduit notamment par une série de projets visant à rendre médecins et cadres soignants responsables de budgets globaux délégués au niveau des services. En dépit de résultats peu probants, cette réforme sera étendue à tout le système au début des années 1990 lors de la création d'un quasi-marché10. Le Livre blanc Working for Patients ${ }^{11}$ forme la base de la loi de 1990, le NHS and Community Care Act, et contient la réforme la plus radicale de la période, qui s'inscrit pleinement dans le mouvement de nouvelle gestion publique. Par-delà la maitrise des coûts, elle vise à améliorer le rapport qualité/prix du service par la mise en concurrence des établissements hospitaliers. La réforme est effectuée au nom du patient, qui doit être placé au cœur du système et exercer des choix. En réalité, seuls les acheteurs, les autorités sanitaires et les généralistes gestionnaires, sont en position de choisir entre plusieurs hôpitaux ou services. La Charte du patient (Patient's Charter) ${ }^{12}$, qui s'inscrit dans le cadre plus large de la Charte du citoyen (Citizen's Charter), détaille les droits existants des patients et leur accorde de nouvelles garanties. Sous couvert d'une amélioration de la qualité, elle permet d'imposer de nouveaux indicateurs de performance aux établissements. Dans la même veine, l'évolution des fonctions des organismes de scrutation comme l'Audit Commission et l'obligation qui est faite aux médecins et professions soignantes de mener des audits cliniques ${ }^{13}$ s'ajoutent à la panoplie des mécanismes de surveillance dont le centre dispose vis-à-vis du niveau local.

7 Le quasi-marché entraîne enfin un raccourcissement de la ligne hiérarchique qui relie les nouveaux hôpitaux (NHS Hospital Trusts) et le pouvoir exécutif central, le National Health Service Executive (NHSE) ${ }^{14}$. Les échelons intermédiaires sont marginalisés ou même supprimés. Lors de leur transformation en acheteurs, les districts passent d'une position de supériorité hiérarchique vis-à-vis des hôpitaux à une relation contractuelle horizontale. Ce faisant, ils perdent une partie de leur personnel et de leurs anciennes fonctions et leur budget est dorénavant directement versé par le NHSE. Quant à l'échelon régional, il est définitivement supprimé en 1996 et remplacé par des bureaux régionaux (regional offices), des postes décentralisés du pouvoir exécutif central sans existence autonome $\mathrm{e}^{15}$. Une fois les régions absorbées par le NHSE, plus rien ne sépare les établissements hospitaliers du niveau central.

8 Un point essentiel de la réforme est la transformation des hôpitaux publics en Trusts, ou fondations semi-privées, un processus qui sera achevé en l'espace de cinq ans. Ce 
nouveau statut permet aux établissements d'employer directement leur personnel et de créer de nouveaux postes. Il leur accorde surtout une grande autonomie financière, dont le droit de vendre et d'acquérir des biens, d'établir des contrats avec les acheteurs et de générer des revenus propres en provenance du public et du privé. En dépit des apparences, cette dévolution est limitée. Les Trusts font toujours partie du secteur public et à ce titre leur existence légale peut être révoquée par le gouvernement central ${ }^{16}$. Soumis à toute une série d'indicateurs de performance, ils doivent également rendre des comptes annuels au NHSE par l'intermédiaire des nouveaux bureaux régionaux. Même la composition de leur équipe directoriale doit être approuvée par le ministère.

9 La dévolution gestionnaire des années 1980 et 1990 s'accompagne donc dans le secteur hospitalier d'une forte centralisation du service, caractérisée par la mise en place de nouvelles formes de contrôle de l'activité et la marginalisation des échelons administratifs intermédiaires. Dans le cas de la médecine de ville, la déconcentration des pouvoirs est moins ambiguë. Un nouveau statut (fundholder), créé dans le cadre de la loi de 1990, permet aux cabinets de généralistes de gérer leur propre budget, d'établir des contrats d'achat avec les hôpitaux et de réinvestir les économies éventuellement réalisées dans leurs cabinets. Cette réforme associe des principes clés de la nouvelle politique de gestion publique: délégation gestionnaire, responsabilisation des acteurs locaux et mécanismes de marché. La réforme connaît un succès rapide puisqu'en 1996, 57\% de la population anglaise avait pour médecin traitant un fundholder ${ }^{17}$. En acheteurs avertis ${ }^{18}$, les généralistes gestionnaires se regroupent d'abord en agences (commissioning agencies), puis en larges fédérations (multi-funds) ${ }^{19}$, afin d'être en position de force pour négocier des contrats de soins groupés auprès des hôpitaux.

10 En 1993, un projet de contrats globaux, le Total Purchasing Pilots (TPPs), permet à certains généralistes gestionnaires d'acheter tous les soins dont leurs patients ont besoin, y compris ceux réservés jusqu'à cette date aux autorités sanitaires comme les urgences, la psychiatrie interne, les soins de maternité et les prestations spécialisées. À l'origine, le projet avait pour but d'accroître l'autonomie des fundholders mais la notion d'un partenariat entre médecins et autorités sanitaires qui permettrait d'élaborer des stratégies communes émerge rapidement ${ }^{20}$. Dans un cas comme dans l'autre, la marge de manœuvre des autorités sanitaires se trouve diminuée. Vers la fin de la période, les généralistes qui n'ont pas voulu, ou pas pu, opter pour le statut de fundholder, sont autorisés à se regrouper en fédérations alternatives (alternative commissioning groups) afin de pouvoir également négocier des contrats groupés par l'intermédiaire de leurs autorités de tutelle ${ }^{21}$. Comme dans le secteur hospitalier, le transfert de la relation contractuelle vers les cabinets de généralistes forme une pièce essentielle du quasimarché. S'ils avaient été réélus en 1997, les conservateurs auraient accéléré ce mouvement en autorisant tous les généralistes qui le souhaitaient à se regrouper en très grands cabinets de soins (supersurgeries), voire en petits établissements hospitaliers

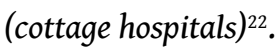

\section{Le projet du New Labour : la régulation indirecte}

11 Dans le domaine sanitaire, comme dans d'autres services publics, on distingue plusieurs tendances dans la politique travailliste depuis 1997. Les deux premières années sont 
caractérisées par une maîtrise des dépenses stricte, qui reflète l'engagement électoral des travaillistes de respecter les plafonds draconiens fixés aux dépenses publiques par les conservateurs avant leur départ. La concurrence étant rejetée au profit d'une «troisième voie » fondée sur le partenariat et la performance ${ }^{23}$, les contrats d'achat de soins sont remplacés par un système de commandes (commissioning) reconductibles pendant trois ans. Au nom de l'efficacité, le nouveau gouvernement maintient néanmoins la division opérationnelle du service entre acheteurs et producteurs, le statut de Trust des hôpitaux et le transfert de pouvoirs vers les cadres locaux. Dans le secteur de la médecine de ville, le statut de fundholder est supprimé et remplacé par de nouvelles structures décentralisées, les groupes de soins primaires (Primary Care Groups).

Tout comme celle des conservateurs, la rhétorique travailliste souligne l'importance de constamment améliorer le service et de l'organiser autour du patient et non pas des professionnels. La différence se trouve dans la volonté d'homogénéiser les conditions d'accès aux soins et leur qualité partout dans le pays afin de rétablir la dimension nationale du service, mise à mal par le marché interne. Les normes de qualité locales doivent ainsi refléter celles déterminées par le centre. Au niveau national, le Livre blanc de 1997 annonce la création d'agences indépendantes qui inspecteront les établissements (Commission for Health Improvement) et jugeront de l'efficacité des médicaments et traitements (National Institute for Clinical Excellence). L'apparente confiance accordée au secteur public pour mener à bien la réforme sanitaire est limitée par la prolifération de formes de contrôle central qui trahissent en définitive une méfiance du gouvernement vis-à-vis des acteurs locaux, les médecins et autres professionnels soignants. Cette tendance s'intensifie en 2000 avec la publication du Livre blanc The NHS Plan $^{24}$ qui annonce la mise en route du régime d'inspection et l'établissement de nouvelles normes cliniques :

Des normes nationales codifieront le traitement de toutes les principales pathologies. L'utilisation des médicaments et traitements appropriés qui auront fait la preuve de leur efficacité et de leur bon rapport qualité/prix sera généralisée dans le pays. D'une façon croissante, les médecins, infirmières et autres soignants exerceront leur activité dans le cadre de protocoles de soins normatifs. Toutes les organisations du NHS seront inspectées de façon indépendante ${ }^{25}$.

13 Pour certains, ce système de surveillance indirecte, relayé à tous les niveaux du service par la structure managériale héritée des conservateurs, traduit une volonté de fort dirigisme gouvernemental ${ }^{26}$. Face à ces critiques, le gouvernement répond par la notion d'« autonomie méritée " (earned autonomy) pour les établissements qui atteignent les objectifs fixés par le centre et, à partir de 2001, développe un discours sur le « localisme » qui vise à promouvoir la participation citoyenne dans l'orientation et la gestion des services publics. Les fondations de soins primaires forment un exemple de mise en œuvre de ce nouveau courant décentralisateur.

\section{Origine et structure des Primary Care Trusts}

Dès 1997, les travaillistes avaient décidé de mettre en place une alternative au système des fundholders ${ }^{27}$. En 1999, 481 groupes de soins primaires ${ }^{28}$ rassemblant tous les médecins, infirmières et autres personnels soignants du secteur de ville sont établis pour une durée initiale de deux ans avec pour mission principale de développer l'offre de soins locale. La réforme doit permettre de modifier l'équilibre des pouvoirs entre le 
secteur de ville et l'hôpital, dominé par la caste puissante des médecins spécialistes (consultants), et de mieux prendre en compte la « voix » des usagers au niveau local.

Bien que les textes officiels soulignent l'aspect innovant d'une réforme qui se veut distincte du modèle étatique des années 1960-70 et de l'approche concurrentielle de la nouvelle gestion publique, on est frappé de constater la continuité par rapport à la période conservatrice. Tout comme les fédérations de généralistes (multi funds), les PCGs bénéficient d'un transfert de pouvoirs du niveau administratif supérieur, les autorités sanitaires ${ }^{29}$, qui leur permet de gérer un budget limité, de décider de l'utilisation de leurs ressources dans le cadre du Programme d'amélioration de la santé (Health Improvement Programme) local et de passer directement des commandes de soins avec le secteur hospitalier ${ }^{30}$.

D'après le Livre blanc de 1997, le processus de transfert des pouvoirs se découpe en plusieurs stades successifs. Au premier niveau, les groupes aident l'autorité sanitaire à passer des commandes de soins; au niveau suivant, ils gèrent leur propre budget ; au niveau 3, les groupes deviennent des organismes quasi autonomes qui traitent directement avec le secteur hospitalier ; enfin, au dernier niveau, ils se transforment en fondations responsables de tout le secteur primaire de leur localitée ${ }^{31}$. Tous les médecins généralistes sont tenus de rejoindre un groupe local avant avril 1999, date à laquelle le Health Act autorise les structures les plus développées à fusionner localement et à solliciter immédiatement le statut de fondation ${ }^{32}$. En 2002, tous les groupes restants deviennent automatiquement des fondations. Globalement, ces nouvelles structures sont responsables de plus de $75 \%$ des dépenses sanitaires locales en $2003^{33}$, une part plus importante du budget du NHS que sous le système des fundholders.

Les Primary Care Trusts dispensent la quasi-totalité des soins au niveau local et gèrent un budget annuel global qui couvre les commandes de soins, les coûts de prescription, les locaux et le matériel informatique. Elles ont pour mission d'évaluer les besoins sanitaires de la population locale, de développer une offre de soins adaptée et de diminuer les inégalités devant la santé. Statutairement, elles doivent établir des partenariats avec les services sociaux et associer les usagers au processus d'achat et d'évaluation des soins. Chaque fondation est gouvernée par un conseil exécutif (Professional Executive Committee) composé de praticiens, de cadres gestionnaires et d'un directeur général (chief executive). Suivant le modèle du privé, cet organe est contrôlé par un conseil d'administration (Trust Board) formé en majorité de directeurs sans pouvoir exécutif (non executive directors) (NEDs) qui doivent, tout comme le Président (lay chairman) ${ }^{34}$, être extérieurs à l'organisation et aux professions médicales et soignantes. Les réunions du conseil d'administration, qui prennent place tous les deux mois, doivent être publiques. Quant au conseil exécutif, il se réunit plus fréquemment et en privé.

Nous allons à présent nous interroger sur le niveau de déconcentration des nouvelles fondations par rapport aux échelons administratifs supérieurs, les autorités sanitaires et le ministère.

\section{L'autonomie sous contrôle des Primary Care Trusts}

Pour certains, les Primary Care Trusts «...incarnent le principe visant à transférer davantage de contrôle et de pouvoirs décisionnels aux praticiens qui sont en contact direct avec le public et ont la charge d'organiser et de dispenser les soins... $»^{35}$. Dotées d'un budget global, libres 
d'employer leur personnel et de déterminer leurs orientations stratégiques, les fondations sont pourtant soumises à diverses formes de régulation: le contrôle hiérarchique, les normes nationales et locales, l'inspection et l'obligation de former des partenariats. Seule la première catégorie constitue une forme de contrôle managérial direct.

20 Tout d'abord, les fondations rendent régulièrement des comptes à leurs supérieurs hiérarchiques directs, les Strategic Health Authorities (SHA), qui surveillent de près leur performance financière et clinique et peuvent, en cas de problème grave, forcer une équipe de direction à démissionner. Les normes prescriptives et les objectifs sanitaires nationaux constituent une autre forme de régulation qui connaît un succès croissant depuis 1997. Au niveau national, des protocoles de soins (National Service Frameworks) déterminent le traitement médical à suivre pour la plupart des pathologies et les décisions du National Institute for Clinical Excellence réduisent les choix en matière de prescription médicale. Localement, la mise en œuvre de ces normes est contrôlée par le biais d'indicateurs de performance informatisés, dont les résultats sont transmis aux niveaux supérieurs par la voie hiérarchique. Les fondations sont par ailleurs tenues de signer des accords de service par secteur (service agreements) avec les hôpitaux et les autorités sanitaires qui incluent toute une série de critères de qualité, d'objectifs et de mécanismes d'évaluation. Loin de se cantonner aux aspects purement organisationnels $\mathrm{du}$ service tels que la durée d'attente maximale pour une première consultation avec un spécialiste, les objectifs s'intéressent de plus en plus à des résultats cliniques mesurables (health outcomes) comme la prévention de certaines pathologies et le taux de mortalité par groupe de patients ${ }^{36}$.

21 Les fondations sont également régulées par l'agence nationale d'inspection (Commission for Health Improvement) ${ }^{37}$ qui les évalue en fonction de leur performance clinique, organisationnelle et financière. À la suite de l'inspection, chaque fondation reçoit une note $^{38}$ et fait l'objet d'un rapport qui est rendu public. Enfin, les fondations sont régulées transversalement par l'obligation statutaire de former des partenariats. Depuis 1997, il s'agit là d'une priorité pour le gouvernement, qui considère que seule la coopération trans-sectorielle locale entre le secteur public, privé et associatif (joined-up governement) permettra d'améliorer la qualité du service pour les usagers. Comme les progrès tardaient à venir, le NHS Plan de 2000 a durci le ton et renforcé la dimension prescriptive de ces partenariats : "Le système de santé et les services sociaux doivent être organisés en fonction des besoins du patient, et non l'inverse. Le Service national de santé mettra en place des partenariats et des coopérations à tous les niveaux. " $^{39}$

Tenues d'inclure des représentants des services sociaux dans leurs comités de direction, les fondations doivent également former des partenariats avec ce secteur et diverses organisations bénévoles et privées dans le cadre du programme d'amélioration de la santé local et de programmes nationaux tels que le Joint National Priorities Guidance, qui vise à établir des stratégies communes dans des secteurs prioritaires comme la réduction des listes d'attente et des inégalités devant la santé, et les Joint National Service Frameworks, qui établissent des protocoles de soins communs par pathologie ou groupe de patients. Le plus souvent, la formation de partenariats est la condition sine qua non imposée à l'obtention de fonds réservés, comme ceux de la Health Action Zone Initiative. Par delà la recherche d'une plus grande efficacité, l'obligation de collaborer avec d'autres secteurs est une forme indirecte de régulation centrale qui vise à limiter l'autonomie des professions sanitaires et sociales. 

grande participation citoyenne. Dans quelle mesure les fondations reflètent-elles cette préoccupation du pouvoir central, qui a trouvé un nouveau souffle depuis 2001 avec la notion de «localisme»?

\section{Le lent développement de la démocratie participative}

Historiquement dominé par la profession médicale et caractérisé par une hiérarchie bureaucratique, le NHS implique peu les patients dans l'organisation du service et les choix de traitement. La rhétorique des années 1980 et 1990 sur le pouvoir du consommateur et les droits du patient, qui prétend modifier cet état de fait par les mécanismes du marché, sera transformée par les travaillistes en discours sur la participation citoyenne. Pour les fondations de soins primaires, il s'agit de dépasser le stade de la consultation pour atteindre celui de : «l'implication précoce, systématique et continue du public $»^{40}$ et de "...travailler avec les populations locales pour développer des objectifs et projets communs afin d'améliorer la santé et le bien-être au niveau local $»^{41}$. Compte tenu de l'importance accordée à cette ambition, qui sera soulignée au fil des années, on peut se demander quelles formes de participation du public ont été mises en place par les fondations.

Tout d'abord, le statut des fondations les oblige à informer la population locale et à inclure des représentants du public dans leurs structures. En pratique, le droit à l'information se traduit par la publication des rapports d'activités des fondations et de leurs comptes rendus d'inspection par la Commission for Health Improvement et par le droit pour le public d'assister - mais non de participer - aux réunions du comité d'administration. Pour ce qui est de la représentation des usagers, deux mécanismes sont prévus. D'une part, le Forum des patients (Public Involvement Forum) ${ }^{42}$ local, défenseur de l'intérêt collectif, a le droit d'envoyer des représentants à ces réunions. D'autre part, les fondations doivent recruter les directeurs non exécutifs parmi la population locale et s'assurer de leur représentativité démographique et sociale. Dans les faits, ce dernier point est rarement respecté, les femmes, les jeunes et les minorités ethniques étant fortement sous-représentés. Par ailleurs, les représentants des usagers n'ont pas le droit de vote lors des réunions, ni même parfois le droit à la parole, ce qui limite sérieusement leur rôle.

La participation active du public au choix, à l'achat et à l'évaluation des soins est encore moins apparente. Dans les années 1990, le développement du marché interne s'était accompagné d'un discours sur l'importance d'associer les usagers à chaque étape de ce processus - de l'identification des priorités à la spécification des services achetés et à leur évaluation ${ }^{43}$. En réalité, seules quelques autorités sanitaires avaient organisé des réunions publiques, des groupes de travail ou des enquêtes par questionnaire à cet effet. Le même manque d'enthousiasme se retrouvait à l'époque chez les fundholders ${ }^{44}$, qui consultaient rarement leurs patients avant d'établir des contrats d'achat de soins. La situation n'a guère évolué depuis l'avènement des fondations. Les médecins généralistes ont gardé un quasi monopole sur le processus de commissioning et leur statut indépendant empêche les comités de direction de leur imposer des changements autoritaires ${ }^{45}$. D'une façon générale, la participation du public prend souvent la forme d'une information plutôt que d'une véritable consultation, une habitude qui s'explique par la culture administrative du NHS d'après un directeur non exécutif : 
Les fondations ont fait un gros effort pour impliquer le public. Les managers du NHS sont bien gentils, probablement parce que ce ne sont pas des managers mais des administrateurs. Quand ils ont un problème, ils trouvent une solution et ensuite ils consultent le public. Et bien, ce n'est pas de la consultation, c'est de l'information. ${ }^{46}$

Les comités de direction restent dominés par les médecins et autres professionnels de la santé, qui décident du niveau de participation du public. Le plus souvent, celle-ci se limite aux obligations statutaires qui prennent la forme de réunions publiques, consultations ponctuelles et enquêtes de satisfaction auprès des usagers. Dans l'immense majorité des cas, le public continue d'être exclu du processus de sélection et d'achat des services hospitaliers. Or, les enjeux sont déterminés à ce stade durant lequel les choix entre plusieurs hôpitaux ou services, voire les décisions d'exclure certains traitements en faveur d'autres, sont effectués.

Le problème d'une véritable représentation démocratique reste entier. Les porte-parole officiels des patients siégeant aux réunions des comités de direction des fondations, tout comme ceux sollicités pour participer à des partenariats, ont tendance à être un peu toujours les mêmes et à représenter des groupes d'usagers spécifiques plutôt que la population dans son ensemble.

\section{Conclusion}

Les années 1980-90 se caractérisent par un double mouvement de déconcentration gestionnaire et de renforcement du contrôle central sur le niveau local. Le transfert de responsabilités aux établissements dans les années 1980 s'inscrit dans le cadre de réformes managériales qui accroissent le pouvoir du niveau national. Le passage à un système de marché au début des années 1990, qui se caractérise notamment par la mutation des autorités sanitaires et de certains médecins généralistes en acheteurs locaux, accélère le mouvement de déconcentration gestionnaire mais affaiblit les niveaux intermédiaires, ce qui entraîne un raccourcissement de la ligne hiérarchique entre le centre et le niveau local. En dépit de la rhétorique officielle sur les droits de l'usager, la réforme ne se traduit pas par une meilleure prise en compte de la "voix » du patient. L'élection du New Labour en 1997 s'accompagne d'un nouveau discours sur la gouvernance en réseaux et la participation citoyenne dans les services publics. En pratique, le transfert de pouvoirs gestionnaires dans le cadre d'un marché de la santé local est conservé, mais il s'accompagne de nouvelles formes de régulation indirectes qui renforcent le dirigisme central. En revanche, le fort degré d'autonomie des nouvelles fondations de soins primaires, leur mode de fonctionnement en partenariat avec d'autres secteurs et l'obligation qu'elles ont d'associer le public à leurs décisions pourraient faire penser qu'elles forment un exemple type de la nouvelle gouvernance et du « localisme » prônés par le gouvernement.

Dans les faits, le niveau régional, et surtout national, exercent un fort pouvoir de contrôle gestionnaire et d'orientation stratégique qui limite leur marge de manœuvre. Si la formation de partenariats avec d'autres organisations du secteur public, privé et associatif caractérise le mode de fonctionnement des Primary Care Trusts, il s'agit le plus souvent d'associations prescrites par le centre qui ne survivront pas forcément à un changement d'orientation politique à ce niveau. En accord avec leurs statuts, les fondations ont ouvert leurs comités de direction au public et aux porte-parole des 
patients, mais cette représentation ne s'est pas pour l'instant accompagnée d'une véritable participation citoyenne. Face à la résistance de la profession médicale, les fondations peinent à impliquer le public dans le processus complexe de choix, d'achat et d'évaluation des services. À terme, la nouvelle gouvernance associée à des mécanismes de régulation régionaux et nationaux devrait permettre de donner plus de pouvoir aux usagers mais ce processus risque d'être encore plus lent que la mise en place de partenariats trans-sectoriels.

31 En 2005, la mutation graduelle de certains hôpitaux en fondations autonomes (foundation hospitals) présente un nouveau défi pour les Primary Care Trusts qui vont devoir passer d'un système de commandes de soins à un mécanisme de contrats commerciaux négociables avec ces nouveaux producteurs, plus puissants et mieux organisés qu'eux. Tout laisse à penser que des fusions locales de Primary Care Trusts prendront place et que le processus d'achat des soins remontera d'un échelon, au niveau de ces nouvelles organisations ou des autorités sanitaires. Cette évolution risque de rendre encore plus problématique la participation citoyenne au niveau local.

\section{BIBLIOGRAPHIE}

BURNS, Dany ; HAMBLETON, Robin \& HOGGETT Paul. The Politics of Decentralisation. London: Macmillan, 1994, 304 p.

COMMISSION FOR HEALTH IMPROVEMENT. 'What CHI has found' in Primary Care Trusts : Sector Report, 2004.

DAWSON, Sandra \& DARGIE, Charlotte. 'New Public Management: an assessment and evaluation with special reference to UK health'. Public Management Review, vol. 1, nº4, 1999, pp. 459-481.

DEPARTMENT OF HEALTH. The New NHS : Modern, Dependable. London: Department of Health, 1997.

DEPARTMENT OF HEALTH. The New NHS : Modern, Dependable - Developing Primary Care Groups. London: Department of Health, 1998.

DEPARTMENT OF HEALTH. Patient and Public Involvement in the New NHS. London: Department of Health, 1999.

DEPARTMENT OF HEALTH. The NHS Plan : a Plan for Investment, a Plan for Reform. London: Department of Health, 2000.

DEPARTMENT OF HEALTH. Delivering the NHS plan : Next Steps on Investment, Next Steps on Reform. London: Department of Health, 2002.

GLENDINNING, Caroline. 'GPs and Contracts : Bringing General Practice into Primary Care', Social Policy and Administration, vol. 33, n², 1999, pp. 122-123.

HARRISON, Stephen \& POLLITT, Christopher. Controlling Health Professionals : The Future of Work and Organization in the NHS. Buckingham: Open University Press, 1994, 176 p. 
HUDSON, Bob. 'Decentralisation and Primary Care Groups : A paradigm shift for the National Health Service in England?' Policy \& Politics, vol. 27, no 2, 1999, pp. 159-172.

KOGAN, Maurice \& REDFERN, Sally with KOBER, Anémone ; NORMAN, Ian ; PACKWOOD, Tim \& ROBINSON, Sarah. Making Use of Clinical Audit : A Guide to Practice in the Health Professions.

Buckingham: Open University Press, 188 p.

LOCOCK, Louise \&, DOPSON Sue. 'The three in one and one in three : Changing relations between centre and field in the NHS in the UK'. Public Management, Vol. 1, n¹, 1999, pp. 27-47.

McLAUGHLIN, Kate ; OSBORNE, Stephen \& FERLIE Ewan. New Public Management : Current Trends and Future Prospects. London: Routledge, 2002, 355 p.

MILEWA, Timothy ; DOWSWELL, George \& HARRISON, Stephen. 'Partnerships, power and the "new" politics of community participation in British health care'. Social Policy and Administration, vol. 36, nº7, 2002, pp. 796-809.

NHS EXECUTIVE. Primary Care Trusts : Establishment, the Preparatory Period and their Functions. Leeds: Department of Health, 1999.

POLLITT, Christopher ; BIRCHALL, Johnston \& PUTMAN Keith. Decentralising Public Service Management. London: Macmillan, 1998, 211 p.

POLLOCK, Allyson M. NHS plc: The Privatisation of Our Health Care. London: Verso, 2004, 271 p.

STOKER, Gerry. Transforming Local Governance : from Thatcherism to New Labour. Government Beyond The Centre Series. Basingstoke: Palgrave Macmillan, 2004, 250 p.

\section{NOTES}

1. Voir Sandra DAWSON \& Charlotte DARGIE, 'New Public Management: an assessment and evaluation with special reference to UK health', Public Management Review, vol.1, no4, 1999, pp. 459-481.

2. Ewan FERLIE \& Louise FITZGERALD, 'The sustainability of the New Public Management in the UK', in Kate McLAUGHLIN, Stephen OSBORNE, Ewan FERLIE, New Public Management: Current Trends and Future Prospects, London: Routledge, 2002, p. 343.

3. Gerry STOKER, Transforming Local Governance: from Thatcherism to New Labour, Basingstoke: Palgrave Macmillan, 2004, p. 11-12.

4. Voir Dany BURNS, Robin HAMBLETON \& Paul HOGGETT, The Politics of Decentralisation: Revitalising Local Democracy, London: Macmillan, 1994.

5. Voir par exemple Martin MINOGUE, Charles POLIDANO \& David HULME, Beyond the New Public Management: Changing Ideas and Practices in Governance, Cheltenham: Edward Elgar, 1998, p. 5.

6. Dans le NHS, le secteur primaire comprend la médecine générale, les soins infirmiers à domicile (district nursing), le secteur de la pharmacie et de l'optique de ville et certains services hospitaliers de proximité.

7. Voir notamment Ewan FERLIE \& Louise FITZGERALD, op. cit., p. 352.

8. Sauf en cas d'urgence médicale, auquel cas le patient peut se rendre directement à l'hôpital le plus proche.

9. DEPARTMENT OF HEALTH AND SOCIAL SECURITY, The NHS Management Inquiry (The Griffiths Report), Press Release nํ8/30, 1983.

10. Au début des années 1980 , des budgets cliniques (clinical budgets), gérés par les chefs de service et relatifs aux dépenses courantes hormis les salaires, sont testés dans trois autorités sanitaires ; à la suite de Griffiths, des budgets globaux (management budgets) couvrant à la fois les 
dépenses courantes et d'investissement sont à leur tour mis à l'essai dans quatre autorités sanitaires; enfin, la Resource Management Initiative (1986), introduite dans six hôpitaux et plusieurs centres de santé locaux, implique médecins et cadres soignants dans la gestion d'un budget global. Avant même l'évaluation du projet en 1991, le gouvernement avait décidé d'étendre ce système à l'ensemble du NHS. Voir Stephen HARRISON \& Christopher POLLITT, Controlling Health Professionals: The Future of Work and Organization in the NHS, Buckingham: Open University Press, 1994, pp. 82-85.

11. DEPARTMENT OF HEALTH, WELSH OFFICE, SCOTTISH HOME AND HEALTH DEPARTMENT AND NORTHERN IRELAND OFFICE, Working for Patients. Caring for the 1990s, Cmnd. 555, London: HMSO, 1989.

12. DEPARTMENT OF HEALTH, The Patient's Charter: Raising the Standard, Citizen's Charter, London: HMSO, 1991.

13. Voir Tim PACKWOOD \& Anémone KOBER, 'Clinical Audit and managing health systems' in Maurice KOGAN \& Sally REDFERN et al., Making Use of Clinical Audit : A Guide to Practice in the Health Profession, Buckingham : Open University Press, pp. 106-127.

14. Suivant les recommandations de Griffiths (1983), la fonction d'élaboration de la politique sanitaire est séparée de la gestion stratégique et opérationnelle du service. Deux comités de direction sont établis au niveau central - le NHS Supervisory Board, présidé par le ministre de la santé, et le NHS Management Board, dirigé par un directeur général. À la suite de diverses fusions, deux nouvelles structures émergent à la fin des années 1980, le Policy Board et le NHS Executive. Voir Louise LOCOCK \& Sue DOPSON, 'The three in one and one in three: Changing relations between centre and field in the NHS in the UK', Public Management, Vol.1, n¹, 1999, p. 29.

15. Voir Louise LOCOCK \& Sue DOPSON, op. cit., pp. 29-30.

16. Christopher POLLITT, Johnston BIRCHALL \& Keith PUTMAN, Decentralising Public Service Management, London: Macmillan, 1998, p. 53.

17. 'Labour backs GP budgets', The Guardian, 4 December 1996.

18. Voir C. SPOOR \& J. MUNRO, 'Do budget-holding physicians respond to price? The case of fundholding in the UK', Health Services Management Research, Vol.16, no4, 2003, pp. 261-267.

19. En 1996, il en existait 17, qui regroupaient environ deux millions de patients et 350 cabinets. Voir AUDIT COMMISSION, What the Doctor ordered : a Study of GP Fundholders in England and Wales, London: HMSO, 1996.

20. Initialement testé dans quatre sites, le projet fut élargi à 87 sites anglais et écossais entre 1995 et 1997 avant d'être évalué par le King's Fund, un organisme indépendant.

21. Voir Caroline GLENDINNING, 'GPs and Contracts: Bringing General Practice into Primary Care', Social Policy and Administration, vol. 33, n², 1999, pp. 122-123.

22. CONSERVATIVE PARTY, You can only be sure with the Conservatives: The Conservative Manifesto, 1997.

23. '[...] A "third way" of running the NHS - a system based on partnership and driven by performance.' (DEPARTMENT OF HEALTH, The New NHS: Modern, Dependable, Cmnd.3807, London: The Stationery Office, 1997, ch. 2.).

24. DEPARTMENT OF HEALTH, The NHS Plan : A Plan for Investment. A Plan for Reform, Cmnd. 4818, London: The Stationery Office, 2000.

25. 'National standards for treating all the major conditions will have been established. Appropriate drugs and treatments which are shown to be clinically and cost effective will be in use in every part of the country. Doctors, therapists and nurses will increasingly work to standard protocols. There will be independent inspection of NHS organisations.' (The NHS Plan, op. cit., p. 20).

26. Voir par exemple T. FREEMAN, 'Measuring progress in clinical governance: assessing the reliability and validity of the Clinical Governance Climate Questionnaire', Health Services Management Research, Vol.16, n4, 2003, pp. 234-250.

27. EXECUTIVE LETTER EL (97) 37, 1997. 
28. À la suite de fusions, les groupes, transformés en fondations, ne sont plus que 303 en 2003. À l'inverse, la population moyenne représentée par chaque organisation passe de 100000 personnes à 170000 au cours de la même période. Allyson M. POLLOCK, NHS plc: The Privatisation of Our Health Care, London: Verso, 2004, p. 53.

29. En 2002, les District Health Authorities fusionnent avec les Family Health Service Authorities pour former 28 autorités sanitaires stratégiques (Strategic Health Authorities).

30. Bob HUDSON, 'Decentralisation and Primary Care Groups : a paradigm shift for the National Health Service in England', Policy \& Politics, Vol.27, n², 1999, pp. 159-172.

31. DEPARTMENT OF HEALTH, The New NHS : Modern, Dependable, 1997, op. cit., ch. 5.

32. Les premières fondations de soins primaires sont créées en avril 2000 par autorisation spéciale du ministre de la Santé. NHS EXECUTIVE, Primary Care Trusts: Establishment, the preparatory Period and their Functions, Leeds: Department of Health, 1999, p. 1.

33. Allyson M. POLLOCK, op. cit., p. 51.

34. Jusqu'à 2002, le directeur et les membres extérieurs étaient nommés par les autorités sanitaires, par pouvoir délégué du ministre, mais depuis cette date les nominations sont effectuées par la NHS Appointments Commission. NHS EXECUTIVE, Primary Care Trusts: Establishment, the preparatory Period and their Functions, Leeds: Department of Health, 1999, pp. 2-3 et entretien avec un directeur non exécutif, North Oxfordshire Primary Care Trust, avril 2004.

35. '[Primary Care Trusts]... embody the principle of devolving more control and decision-making power to the frontline clinicians involved in delivering and arranging care for patients.' (COMMISSION FOR HEALTH IMPROVEMENT, What CHI has found in Primary Care Trusts. Sector report, 2004, p. 6.)

36. En tant qu'organismes publics, les fondations sont également soumises à diverses directives générales, comme l'obligation d'avoir des comités de direction qui reflètent la diversité ethnique du pays (d'après le Race Relations Amendement Act de 2000).

37. La Commission for Health Improvement est remplacée par la Healthcare Commission en avril 2004.

38. Ou plutôt des étoiles : en fonction de ses résultats globaux, l'établissement reçoit une, deux ou trois étoiles, voire aucune en cas de problème grave.

39. 'The health and social care system must be shaped around the needs of the patient, not the other way round. The NHS will develop partnership and cooperation at all levels of care.' (DEPARTMENT OF HEALTH, The NHS Plan : A Plan for Investment. A Plan for Reform, op. cit., p. 5.)

40. '[expected to put in place mechanisms for] the early, systematic and continuous involvement of the public'. (DEPARTMENT OF HEALTH, The New NHS : Modern, Dependable - Developing Primary Care Groups, London: Department of Health, 1998 in MILEWA Timothy, DOWSWELL George \& HARRISON Stephen, 'Partnerships, power and the "new" politics of community participation in British health care', Social Policy and Administration, vol.36, nำ, 2002, pp. 796-809).

41. '...work with their local communities to develop shared goals and aims for improving local health and well-being'. (DEPARTMENT OF HEALTH, Patient and public Involvement in the New NHS, London, Department of Health, 1999, p. 11).

42. Début 2004, les Public Involvement Forums remplacent les Community Health Councils et reprennent leurs fonctions principales.

43. Voir NHS MANAGEMENT EXECUTIVE, Local Voices, London : NHSME, 1992.

44. En 1996, l'Audit Commission note que seulement $4 \%$ des fundholders consultent leurs patients ou mènent des enquêtes d'opinion avant d'élaborer leurs rapports stratégiques. Voir AUDIT COMMISSION, What the Doctor ordered : a Study of GP Fundholders in England and Wales, London: HMSO, 1996.

45. Les médecins généralistes, tout comme les dentistes, pharmaciens et optométristes, ont des contrats qui précisent leurs obligations de service mais ils ne sont pas directement employés par le NHS. Voir COMMISSION FOR HEALTH IMPROVEMENT, What CHI has found in: Primary Care Trusts. Sector Report, 2004, p.7. 
46. “[...] PCTs have made a big attempt to involve the public. NHS managers are very sweet, largely because they are not managers but administrators. What they do when they have a problem...they come up with a solution, and then they consult the public. Well, that's not consulting, it's informing.' (Entretien avec un directeur non exécutif, North Oxfordshire Primary Care Trust, avril 2004).

\section{RÉSUMÉS}

Dans les années 1980 et 90, les réformes du service national de santé, qui s'accompagnent d'un discours officiel sur le pouvoir de l'usager, se caractérisent par un transfert des pouvoirs gestionnaires en faveur des hôpitaux et des médecins généralistes qui s'inscrit dans le mouvement de la nouvelle gestion publique. Depuis 1997, la rhétorique travailliste se distingue de celle de leurs prédécesseurs par l'importance donnée aux notions de «localisme » et de démocratisation du service mais en pratique de nouvelles formes de régulation indirecte par le centre sont apparues. Cet article s'intéresse au cas des fondations de soins primaires afin de déterminer dans quelle mesure les principes gouvernementaux se traduisent au niveau local par la déconcentration des pouvoirs et la participation active des usagers au service de santé.

In the 80 s and 90s, official rhetoric emphasised the notion of consumer power in the NHS. The reforms which were set up as part of the New Public Management movement were characterised by budgetary and managerial devolution to hospitals and fundholding GPs. Since 1997, New Labour has supported the principles of 'localism' and democratisation in the NHS while in practice new forms of indirect regulation by the centre have appeared. This article studies the case of primary care trusts in order to determine to what extent the government's line has led to greater devolution and user participation at local level.

\section{AUTEUR}

\section{ANÉMONE KOBER-SMITH}

Université de la Sorbonne Nouvelle-Paris III 